\title{
Review
}

\section{Circadian clocks, diets and aging}

\author{
Amol Chaudhari, Richa Gupta, Kuldeep Makwana and Roman Kondratov* \\ Department of Biological, Geological, and Environmental Sciences and Center for Gene Regulation \\ in Health and Diseases, Cleveland State University, Cleveland, OH, USA
}

\begin{abstract}
Diets and feeding regimens affect many physiological systems in the organism and may contribute to the development or prevention of various pathologies including cardiovascular diseases or metabolic syndromes. Some of the dietary paradigms, such as calorie restriction, have many well-documented positive metabolic effects as well as the potential to extend longevity in different organisms. Recently, the circadian clocks were put forward as integral components of the calorie restriction mechanisms. The circadian clocks generate the circadian rhythms in behavior, physiology, and metabolism; circadian disruption is associated with reduced fitness and decreased longevity. Here we focus on recent advances in the interplay between the circadian clocks and dietary paradigms. We discuss how the regulation of the circadian clocks by feeding/nutrients and regulation of nutrient signaling pathways by the clocks may contribute to the beneficial effects of calorie restriction on metabolism and longevity, and whether the circadian system can be engaged for future medical applications.
\end{abstract}

Keywords: Biological rhythms and clocks, cell signaling, transcription, food anticipation

\section{Introduction}

The 24-hour rhythms in behavior, physiology and metabolism are known as the circadian rhythms. Many of these rhythms are regulated by internal physiological systems known as circadian clocks [1]. It is believed that circadian clocks evolved as an adaptation to periodic light/dark changes caused by the Earth's rotation to provide organism's synchronization with the environment and coordination of multiple metabolic processes within the organism [2]. Circadian clocks have been found in different organisms from unicellular to mammals including humans. In mammals, almost all physiological systems such as cardiovascular, nervous, digestive and urinary systems demonstrate some sort of circadian control and rhythms [3, 4]. The importance of circadian clocks and rhythms for human health

\footnotetext{
*Corresponding author: Roman Kondratov, Department of Biological, Geological, and Environmental Sciences and Center for Gene Regulation in Health and Diseases, Cleveland State University, Cleveland, OH 44115, USA. Tel: +1 216523 7199; E-mail: r.kondratov@csuohio.edu.
}

has been demonstrated in multiple studies: circadian disruption significantly disturbs homeostasis for many physiological processes and might lead to the development of pathological states. For example, epidemiological data on shift workers show that the risk of metabolic, cardiovascular diseases and cancer is significantly increased in shift workers in comparison with the general population $[2,5,6]$. Animal models also support the importance of the clock: mice with targeted disruption of clock genes, in addition to the disrupted rhythms in behavior and physiology, demonstrate a predisposition to the development of a variety of pathologies [7]. Over the last decade a significant body of evidence accumulated on the interconnections between circadian clocks and aging [8]. Aging causes deterioration of multiple physiological systems and is considered as a single high-risk factor for the development of diseases such as cancer, diabetes, neurodegeneration, osteoporosis and sarcopenia [9]; interestingly, circadian clock disruption is considered to contribute to the development of these pathologies [2, 10-12]. Understanding the molecular mechanisms of aging is important for 
prevention and treatment of pathologies. Multiple signaling pathways and physiological systems have been proposed as regulators of aging process, but mechanisms of aging are still not quite known. Circadian clocks might be one of these systems [13]. Indeed, data from animal models suggest that circadian disruption through genetic ablation of several circadian clock components results in reduced lifespan and numerous pathological changes [7]. On the other hand, it was proposed that increased robustness of the circadian rhythms can positively affect longevity. Studies on alpha MUPA transgenic mice revealed that they live longer compared to wild-type mice and show high amplitude circadian rhythms in clock gene expression in the liver and clockcontrolled output systems [14].

Synchronization of physiological processes with the environment is also important: indeed, experimental jet lag or exposure of animals to artificially short or long light/dark cycles causes reduction in lifespan [8, 13]. The period of the internal clock rhythms might also be important; for example, mice with the internal circadian period close to 24 hours live longer than mice whose internal circadian periods are significantly different from 24 hours. How circadian clocks may regulate aging? Circadian clocks control the oxidative stress, cell cycle, cell death and DNA damage response; all of these systems have been implicated in the control of aging and aging-associated pathologies $[13,15]$. There are several excellent reviews on this subject; therefore, here we will discuss only recently emerged data on how the interplay between dietary regimens, nutrient sensing/response pathways and circadian clocks may affect health and lifespan in different model organisms.

\section{The circadian clocks}

The circadian clocks are entrained by the light and generate daily rhythms in locomotor behavior; these rhythms persist in constant darkness (without light cues) for several months [1]. The central, or master circadian oscillator is located in the suprachiasmatic nucleus ( $\mathrm{SCN}$ ) of the anterior hypothalamus. The SCN clock is entrained by the light/dark cycle (light-entrainable pacemaker) and is synchronized with the environmental light/dark cycle (entrainment) through signals from the retina [16]. SCN ablation results in the disruption of circadian rhythms in locomotion. Other organs and tissues have their own circadian clocks known as peripheral clocks [17].
Circadian clocks operate literally in every cell, and circadian rhythms can be detected at the level of an individual cell as rhythms in gene expression. The peripheral clocks generate rhythms in gene expression and different physiological outputs such as body temperature, blood pressure, hormone secretion and metabolic activities [2, 3, 17]. Peripheral clocks are regulated by the SCN master clock, but many other cues, including feeding, also affect the clocks and might uncouple the central and peripheral clocks. The circadian clock integrity depends on activities of several genes, the so-called core circadian clock genes, which are expressed in the SCN and other peripheral tissues. These genes and their products form interconnected transcription-translation feedback loops [18, 19] (see Fig. 1). Therefore, expression and activity of circadian clock proteins are interconnected. Two proteins containing basic helix-loop-helix (bHLH) PAS (Period, ARNT, SIM) domains, Brain, and Muscle ARNT Like 1 (BMAL1) and Circadian Locomotor Output Cycles Kaput (CLOCK) are the positive regulators of transcription. In some tissues such as forebrain and cardiovascular system the closest homologue of CLOCK - NPAS2 substitutes for CLOCK. The BMAL1:CLOCK or BMAL1:NPAS2 complexes bind to the circadian E-box elements located in the promoter region of various genes and activate expression of circadian clock genes such as the three Periods (Per1, Per2 and Per3), two Cryptochromes (Cryl and Cry2), the nuclear receptors $R e v-E r b \alpha$ and $R e v-E r b \beta$, and the retinoid-related orphan receptors (RORs). Negative elements of the loop, the PER and CRY proteins, form complex in the cytoplasm after translation, translocate to the nucleus and act as the negative regulators of the BMAL1:CLOCK complex, thus inhibiting their own expression and completing the negative feedback loop. REVs and RORs compete for binding to the ROR elements in the promoter of Bmall, with RORs being a positive regulator of Bmall transcription and REVs being a negative regulator, and form a second positive-negative feedback loop [19]. Circadian clock proteins undergo multiple posttranslational modifications such as phosphorylation, sumoylation, and acetylation, which provides an additional level of regulation for the core circadian clock gene expression [20]. The circadian clock controls cellular processes and metabolism through the regulation of the ClockControlled Genes (CCGs). The circadian regulation of CCGs expression occurs via BMAL1:CLOCK and REVs-dependent mechanisms or through the rhythmic activity of clock-controlled transcriptional 


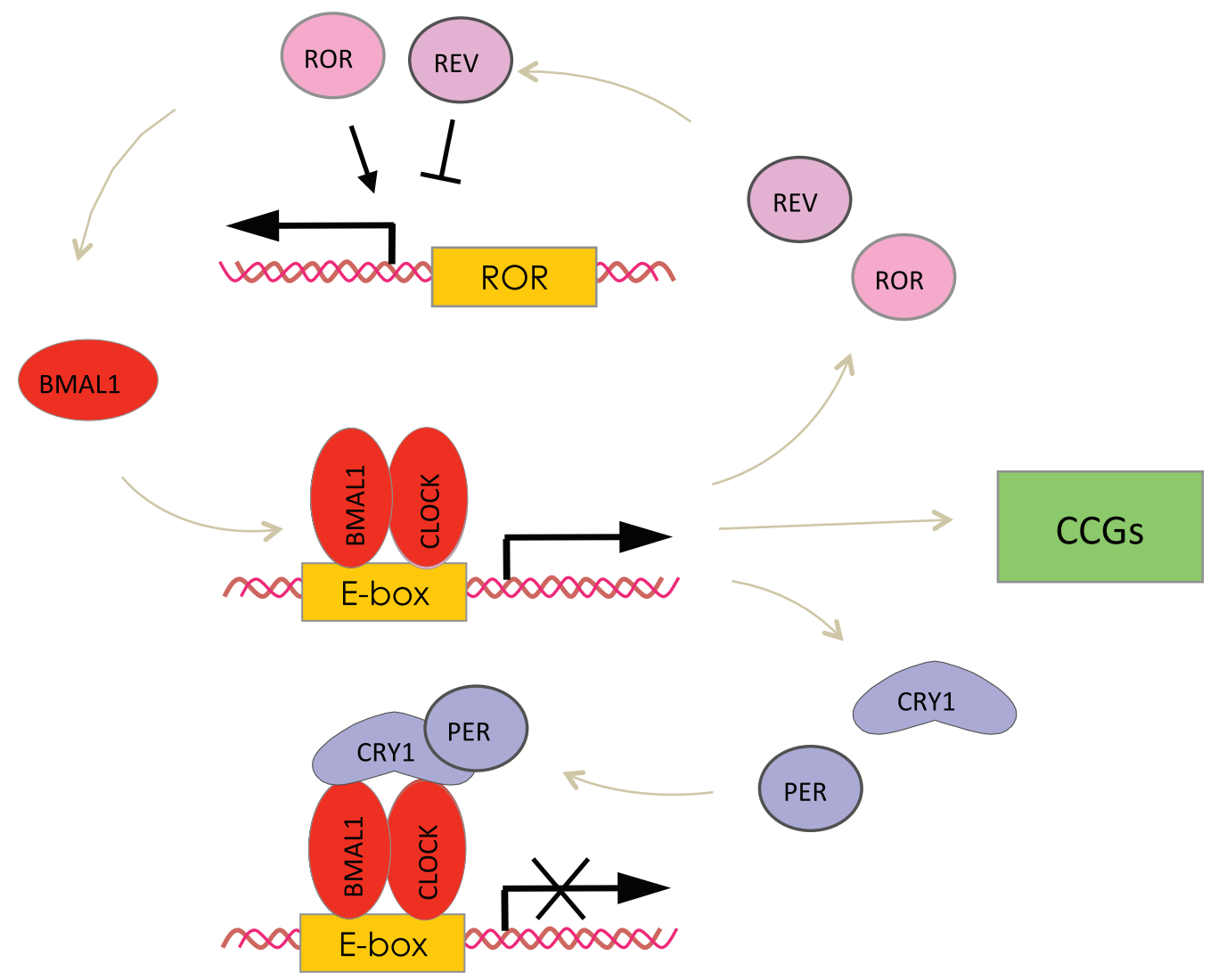

Fig. 1. The molecular circadian oscillator. The basic-HLH-PAS domain containing transcriptional factors BMAL1 and CLOCK (NPAS2) regulate transcription of genes with the circadian E-box elements in the promoter, and represent the positive arm of the transcriptionaltranslational feedback loop. The BMAL1:CLOCK complex activates the expression of Per and Cry genes. PER and CRY represent the negative arm of the loop; they form complexes and inhibit the activity of the BMAL1:CLOCK complex and hence their own expression. Rev-Erbs and RORs represent an additional loop, these transcriptional factors negatively (Rev-Erbs) and positively (RORs) regulate the expression of BMAL1. Finally, the BMAL1:CLOCK complex regulates the expression of circadian clock controlled genes (CCGs), which provide circadian output in physiology.

factors. In every tested tissue about $10 \%$ of transcriptome demonstrates circadian rhythms [21].

In addition to the above-described circadian clocks (the light-entrainable pacemaker in the SCN and peripheral circadian clocks), the existence of other types of circadian clocks have been proposed. Food-entrainable oscillator (FEO) produces food anticipatory activity (FAA) 2-3 hours before the scheduled feeding time. The existence of the foodentrainable peripheral oscillators (FEPOs), which are situated in the peripheral organs was also suggested, FEPOs entrain peripheral circadian clocks upon scheduled feeding [22]. Despite many studies, anatomical location and the mechanisms of FEO and FEPO is not clear [23]. SCN-lesioned rats still can be entrained to scheduled feeding. The result suggested that the SCN is not the site, as these rats still possessed the food anticipatory activity. Many other areas of the brain were studied to identify FEO, but the location of FEO remains unidentified. (Detailed review on studies for the quest of FEO [24])

Light- and food-entrainable clocks may interact with each other; for example, FEO may use the SCN-based clock as a reference clock [23], and the light-entrainable pacemaker may control the period of the food-entrainable pacemaker [25]. Studies also show that clock genes may play a role in the control of the FAA rhythms. Mutation of Per2 ${ }^{\text {Brdm } 1}$ gene leads to attenuation of FAA rhythms [26]. Reports on the role of BMAL1 in the regulation of FAA are controversial. One group reported that the down regulation of BMAL1 expression in dorsomedial hypothalamus (DMH) affected the formation of FAA; however, several other groups reported normal FAA [27]. 
Recently, it was reported that activity of the mechanistic target of rapamycin complex 1 (mTORC1) [28] demonstrates 24-hour rhythms in several tissues in mice. These rhythms can be observed under both ad libitum and time-restricted feeding conditions, and they might be under the control of a clock-like mechanism. Indeed, when the rhythms were entrained by the feeding, they persisted in fasted animals for at least three cycles, suggesting some internal regulatory mechanism. Importantly, the rhythms of mTORC1 activity were preserved in mice with target disruption of CRY genes, and these mice have disrupted circadian rhythms in the SCN-controlled behavior; thus, this clock is not identical with the light-entrainable circadian clock. This hypothetical clock was named NAMO (Nutrient Anticipation Metabolic Oscillator); the molecular pathways responsible for NAMO operation are currently unidentified, and it is also not known if any interaction between NAMO and other circadian clocks exist.

\section{Clocks, dietary paradigms and longevity}

Diet affects many physiological systems including the circadian clocks. In nocturnal rodents, timerestricted feeding during the light phase of the day (unlimited amount of the food provided during the normal rest period) alters the phase of the circadian clock and clock-controlled genes expression in the peripheral tissues, whereas the gene expression in central clock (SCN) is not affected and still under the control of the light/dark cycle [29, 30]. Peripheral clocks adopt to the novel feeding regimens differently: the liver can adapt faster than kidney, lungs or heart. Uncoupling the peripheral and central circadian clocks also cause changes in blood glucose, triglyceride and free fatty acid levels, resembling the state of the metabolic syndrome [29, 30]. All these studies demonstrate that the peripheral clocks can be regulated by the feeding, and support the theory that circadian misalignment has a negative impact on health.

Another common dietary paradigm, which models the Western diet, is the high fat diet. In rodents, the high fat diet leads to obesity and causes multiple metabolic changes. Kohsaka et al. demonstrate that the high fat diet also affects the central and peripheral circadian clocks: the major effect was disruption of circadian rhythms in locomotor and feeding behaviors, and significant reduction of the rhythm ampli- tude of the circadian clock and clock-controlled gene expression in the liver [31]. The high fat diet causes increased blood glucose level, insulin resistance, and many other metabolic changes; circadian clock disruption might be one of the contributing factors [32]. Sherman et al. and Hatori et al. applied time-restricted feeding to animals on the high fat diet $[33,34]$, in contrast to above cited studies where the time-restricted feeding was applied during the light/rest phase, here the time-restricted feeding was applied during the night/activity phase (Hatori et al.) and during light phase (Sherman et al.). In Hatori et al. study, the central and peripheral clocks must be synchronized. Time-restricted feeding restored liver rhythms in circadian clock gene expressions and, importantly, normalized metabolism and reduced obesity-associated inflammation. Noteworthy, the effect of metabolic improvements was achieved without any reduction in food intake [34]. At the same time, Sherman et al. achieved metabolic benefits without synchronization of peripheral and central clocks [33]. A pilot study in humans supports the data generated in the experimental system; as it was reported by Panda and colleagues when overweight people restrict their feeding time to day time they show a reduction in body weight [35]. How the time-restricted feeding improve metabolism is unknown, and whether the clock functions are important for metabolic benefits of the time-restricted feeding needs to be studied.

The long-term effects of time-restricted feeding applied during the day/rest period on health and longevity have not been studied, and whether timerestricted feeding applied during the night/activity period without limitation of food intake would have any beneficial effects for animals on the regular diet is not known and needs to be studied. Calorie restriction is another commonly used dietary paradigm. In mammals, food is provided, in most cases, as a single meal; the food is consumed during few hours; therefore, calorie restriction was frequently used to study food anticipatory activity [36] and was also called time-restricted feeding in many studies. Calorie restriction is known to have multiple metabolic benefits and most importantly, significantly delays aging, reduces the risk of age-associated diseases and increases longevity in many organisms from yeast to mammals [37]. Several studies address the interaction between calorie restriction and the circadian clock. Calorie restriction affects the expression of the circadian clock genes in flies and in mammals. In Katewa et al. study calorie restriction was applied 
to Drosophila; the amplitudes of expression of several clock genes were significantly induced by calorie restriction in the head and total body of flies [38]. In Patel et al. study calorie restriction was applied in mice, food for the calorie-restricted group was provided during the dark phase of the day; therefore, effects on the synchronization of central and peripheral clocks must be minimal [39]. Indeed, as it was expected, no effect on the phase of tested clock gene expression in the liver was detected. Calorie restriction has a significant effect on the amplitude and daily average expression of Bmal1, Per1 and Per2 genes in the liver. Mendoza et al. studied the effect of calorie restriction on the expression of circadian clock genes in the SCN and found that similar to Patel et al. study, calorie restriction significantly affected the expression of several clock genes in the SCN [40]. Thus, calorie restriction regulates circadian clock gene expression in different organisms and in different tissues and might affect both central and peripheral clocks.

Patel et al. and Katewa et al. also investigated if circadian clocks play any role in the calorie restriction effects on metabolism and longevity [38, 39]. Both in Drosophila and mice, disruption of the circadian clock through the knockout of the core clock genes (Per and Tim in Drosophila and Bmall in mice) significantly compromised the beneficial effects of calorie restriction including the effect on longevity. In flies, the circadian clocks are important for calorie restriction-mediated increase in fat turn over. In mammals, calorie restriction mediates reduction in blood IGF-1 level and this effect was compromised in mice deficient for the circadian transcriptional factor BMAL1. Thus, these studies established that calorie restriction and circadian clock mechanisms are interlinked, and raised many important questions that need to be studied: How does calorie restriction affect the clock mechanisms? How does the clock contribute to the beneficial metabolic effects of calorie restriction? Do other lifespan-extending interventions such as treatment with rapamycin, or genetic models of extreme longevity such as Ames dwarf mice, require the clock? Is it possible to extend the lifespan by targeting the clock?

\section{How the diet affects the circadian clocks?}

Oscillation of gene daily expression is regulated by interplay between the circadian clocks and feeding cycles at both transcriptional and translational levels [41]. The feeding/fasting cycle causes multiple changes: concentrations of nutrients and microelements in blood and tissues are changed, different hormones are released; below we will discuss the effect of hormones and nutrients on the central and peripheral circadian clocks.

Feeding results in the release of insulin into the bloodstream; insulin affects many organs and tissues: the liver, skeletal muscles, adipose tissue, and brain are major targets. Insulin was proposed as a candidate mediator that resets peripheral clocks in response to feeding cues [42]. Several lines of evidence support this hypothesis. Insulin induces a rapid response in cultured rat hepatocytes, affecting the expression of Per1, Per2, and Decl genes [43]. The MAPK and PI3K inhibitors are found to block the induction of clock genes, suggesting that MAPK and PI3K are involved in resetting clocks downstream of insulin signaling. Cell culture studies are in agreement with in vivo studies: an injection of insulin elicits a rapid change in Per 2 and Rev-erb $\alpha$ expression and shifts the phase of liver clocks. Clock gene expression is rapidly induced by insulin injection in other insulin-sensitive tissues, such as the muscle and adipose tissues, but not in insulin-insensitive tissues, such as the lungs [44]. Thus, the effect of insulin on the peripheral clocks is tissue-specific; it is still unknown if insulin can regulate central clock in the brain.

Glucose is a well-documented circadian clock regulator. In fibroblasts exposed to high glucose concentration the expression of Perl and Per2 genes is down regulated [45]. In cell culture experiments, the phase and period of the circadian rhythms in expression of clock genes such as Per2 depends on glucose concentration in the media. In vivo glucose affects both central and peripheral clocks. Glucose infusion during the daytime strongly induces expression of Per2 in the SCN and suppresses Per2 expression in the liver [46]; thus, the effects of glucose are also tissue-specific. Glucose availability attenuates photic phase response [47]. Insulin and glucose mutually regulate levels of each other, and some of the in vivo effects of glucose might be the effects of insulin and vice versa; however, cell culture data strongly argue that glucose and insulin have independent effects on the clocks. Several intracellular systems may be involved in the glucose sensing by the clocks. Glucose availability indirectly regulates adenosine monophosphate-dependent protein kinase (AMPK), which phosphorylates and controls stabil- 
ity of the CRY proteins [48]. Transforming growth factor B-inducible early gene (TIEG1) and Vitamin D3 upregulated protein (VDUP1) are glucose responsive immediate early genes and upregulation of TIEG1 and VDUP1 contributes to glucose-dependent clock gene expression [45]. Glucose may also regulate cellular clocks through hexosamine/O-GLcNAc pathway; O-GlcNAcylation of BMAL1 and CLOCK inhibits their ubiquitination and degradation regulating the amplitude and phase of clock oscillation [49].

Several nuclear receptors are potential regulators of the circadian clock by lipids (Reviewed in [50]). The nuclear receptors are transcription factors regulated by hydrophobic molecules such as steroids, vitamins, and fatty acids. Many nuclear receptor genes are expressed in the liver in a circadian manner including three PPAR and two REV-ERB isoforms and two ROR isoforms. REV-ERBs and RORs are components of the circadian molecular oscillator and PPARs can modulate the expression of clock genes, therefore, dietary lipids and their metabolic derivatives can signal to the clock through regulation of the nuclear receptor transcriptional activity [50]. As it was discussed above, the high fat diet disrupts the circadian rhythms in behavior and gene expression, therefore affecting both central and peripheral clocks [31]. How the high fat diet signals to the clocks is unknown; releasing some gastrointestinal tract-derived peptides or bile in response to fat and/or deregulation of PPAR alpha signaling in the liver and other tissues can be one of the mechanisms.

The regulation of the clock by amino acids have not been demonstrated in direct experiments; however, it is possible that the total amino acid level or some specific amino acids affect the clock. One example is phosphorylation of the clock transcriptional factor BMAL1 by S6 kinase [51]. S6 kinase is a downstream target of mTORC1, and mTORC1 activity is regulated by growth factors, including insulin, and by amino acid availability [52]; therefore, the above-discussed rhythms in mTORC1 activity could be a consequence of rhythms in amino acid concentration. Thus, hypothetically, rhythms in amino acids concentration may cause rhythms in mTORC1 activity followed by rhythms in S6 kinase, and through BMAL1 phosphorylation may also contribute to clock resetting.

Importantly, a combination of the nutrients might influence the clocks in different ways than treatment with individual nutrients. The rapid phase-shift in the liver clocks is induced by a combination of carbohy- drate and protein, whereas the application of only proteins, sugar, or oil is insufficient [53]. The liver clocks can be reset by injection of glucose combined with amino acids, but not by either of the nutrients alone [54]. Circadian clock gene expression responds to feeding rapidly; indeed, the expressions of Per2 and Decl genes have been induced in the liver of fasted rat just thirty minutes after re-feeding. Similarly, in mice re-feeding affects the Perl, Per2, Decl and $R e v$-erb $\alpha$ expression in the liver within one hour. These findings indicate that nutrients containing glucose and amino acids induce rapid changes in the expression of clock genes, especially Per 2 and Rev$e r b \alpha$, resulting in a phase-shift [42].

Several other food components also affect circadian clocks. Caffeine contained in food and drinks prolongs circadian locomotor rhythms in Drosophila and mice. Interestingly, the dose of caffeine required to affect circadian rhythms is only $\sim 0.05 \%$, which is equivalent to the dose contained in a coffee drink. It is observed that coffee prolongs circadian activity rhythms in mice under constant darkness. Interestingly, caffeine prolongs the circadian period even in cultured cells [55]. Resveratrol, a polyphenol found in red wine and dietary polyamines, also affect clock gene expression [56]. The high-salt diet was shown to affect circadian gene expression in mice. Studies revealed that consumption of a diet containing $4 \% \mathrm{NaCl}$ for more than two weeks induces phase-advances of clock and clock-controlled genes in peripheral tissues, whereas locomotor activity rhythms, including feeding and drinking behaviors are not affected [57]. Gastrointestinal tract-derived hormones and bile acids can also be involved in clock resetting (Reviewed by [58]).

Recent elegant work of Mukherji et al. placed PPAR alpha, Rev-Erb alpha, and CREB as central players for the shift of peripheral clocks upon time restricted feeding [59]. Fasting induced by RF causes reduction in insulin level, release of glucagon and increased blood concentration of free fatty acids. Free fatty acids affect PPAR alpha, and reduced insulin causes GSK3 beta activation, which in turn cause changes in the expression and activity of Rev-Erb alpha. Rev-Erb alpha affects the expression of Bmall and, in turn, the shifted Bmall expression results in shifted peripheral circadian clocks. CREB-dependent regulation of adrenal corticosterone production affects the shift of peripheral clocks in the muscles and heart.

Regulation of the circadian clocks and rhythms by diet and nutrients is summarized in Fig. 2. 


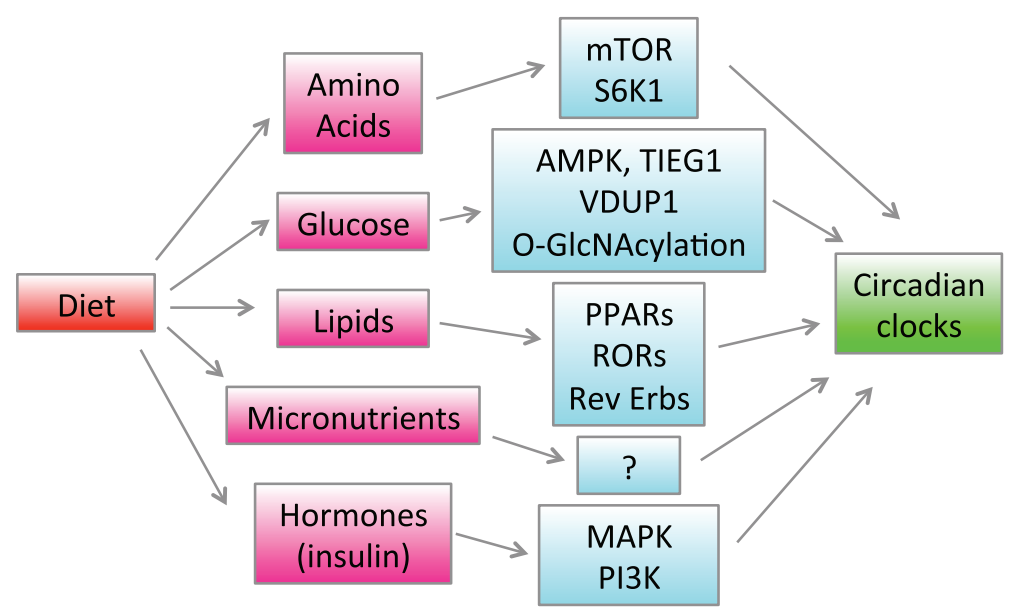

Fig. 2. Regulation of the circadian clocks by diet. Feeding causes changes in blood and tissue concentrations of hormones such as insulin and various nutrients such as glucose, lipids and amino acids. Hormones and nutrients activate different signaling pathways and change gene expression through indicated signaling pathways and transcriptional factors, which in turn affects the circadian clocks in the brain and peripheral tissues.

\section{How the circadian clocks may act as a part of calorie restriction mechanisms}

Calorie restriction leads to several metabolic changes in animals: reduced circulating glucose and insulin levels, increased insulin sensitivity, increased fat turn over, reduced IGF-1 level, decreased activity of mTORC1 signaling pathway, increased activity of sirtuins and increased resistance to various stresses [60-63]. Below we will discuss how the circadian clocks might contribute to all these changes (Illustrated in Fig. 3).

The role of the circadian clocks in glucose homeostasis is well documented and have been extensively reviewed [64]; therefore, we only briefly describe it here. Both central and peripheral clocks are involved in the control of glucose homeostasis, and disruption of synchronization between them has a negative effect on glucose metabolism [64]. Data from animal models of circadian disruption also support the importance of the clock [58]. Blood glucose homeostasis is regulated through external glucose supply (from the digested food), tissue glucose uptake and utilization and gluconeogenesis.

Insulin is the main regulator of glucose uptake and utilization in insulin-responsive tissues. Insulin production and secretion is regulated by the pancreatic clock [65]; disruption of the clock through Clock or Bmal1 knock out compromises the functions of pancreatic islets, which leads to reduced insulin secretion and increased blood glucose level.
At the same time, knock out of Crys or Pers leads to increased insulin level [66, 67]. Insulin sensitivity is also under the control of the circadian clocks, however, the effect of clock disruption on the insulin sensitivity is complicated. While it was proposed that circadian disruption contributes to a reduced insulin sensitivity upon the high fat diet, many circadian clock mutants demonstrate increased insulin sensitivity $[58,68]$. Thus, the effect of the clock on insulin and insulin signaling pathways might be tissue-, time- and gene-dependent, which warrant further study.

Circadian regulation of gluconeogenesis is also complicated. The elements of the negative arm of the circadian feedback loop (Fig. 1) - CRY 1 and CRY 2 proteins - decrease gluconeogenesis by regulating glucagon signaling and CREB phosphorylation [69], and also through regulation of the phosphoenolpyruvate carboxykinase 1 transcription in a glucocorticoid-dependent manner [70]. Another element of the negative loop - PER2 - also decreases gluconeogenesis and promotes glycogen storage by decreasing the activity of glycogen phosphorylase [71]. The clock transcriptional factor Rev-Erb alpha downregulates the expression of many genes important for hepatic gluconeogenesis. On the other side, gluconeogenesis is reduced by deletion of Bmall or Clock mutation [68]. Thus, elements of the positive arm: BMAL1 and CLOCK stimulate gluconeogenesis. Therefore, similarly to the role of the clocks in the insulin signaling, the effect of the clock on glu- 


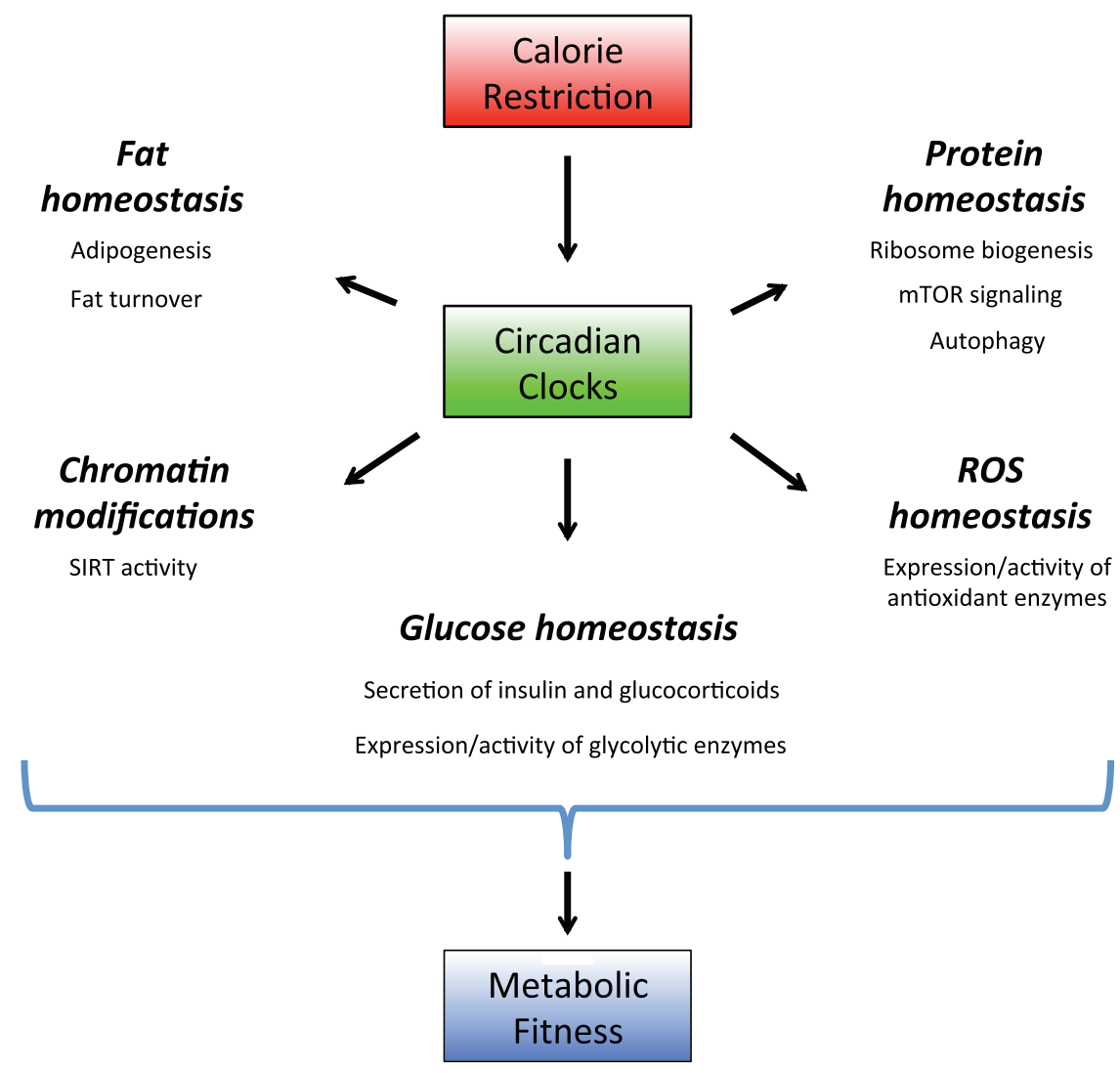

Fig. 3. Circadian clocks as a part of calorie restriction mechanisms. Circadian clocks regulate expression and activity of the rate-limiting enzymes in multiple signaling pathways. Through these regulations the circadian system controls protein, lipid, amino acids and ROS homeostasis. Calorie restriction recruits the circadian clocks, this recruitment leads to optimization of metabolic processes and increases the organism's fitness, which contributes to calorie restriction-mediated longevity benefits.

coneogenesis is tissue- and clock gene-dependent; some mutants have increased and others decreased gluconeogenesis.

The circadian clocks play an important role in regulation of adipose tissue functions and fat turn over. Circadian clock-regulated lipid metabolism pathways in mammals have been revealed by the large-scale profiling, the regulatory circuits involved in fatty acid metabolism have been identified in the adipose tissue, liver, and muscle [72, 73]. Circadian clock disruption results in abnormal fat storage, lipid transport, triglyceride levels, and a decrease in absorption of dietary lipids. Mechanistically, the core components of the molecular clock, REV-ERBs and peroxisome proliferator-activated receptors (PPARs), provide a basis for circadian regulation of lipid metabolism via cyclic transcriptional repression of multiple genes, including the mRNA expression for several rate-limiting lipolytic enzymes [72, 73]. Circadian regulation of adipocyte differentiation occurs through BMAL1 controlled mechanisms and is WNT signaling-dependent $[74,75]$. The circadian clocks are also involved in the regulation of lipid absorption. Clock-controlled deadenylase Nocturnin is central for the circadian dietary fat uptake. In agreement with that, clock mutant mice show altered absorption and transport of diurnal transcription of genes in the small intestine [76]. Finally, adipose tissue-derived hormones including the appetite-suppressant leptin show circadian rhythms [77]. Thus, multiple studies establish a key role of the molecular circadian clock in regulating lipid homeostasis, this regulation is complicated and how it might contribute to the increased fat turn over upon CR needs to be studied. An interplay between the sirtuin pathway and the circadian clocks has also been reported. Sirtuins are NAD+ dependent histone deacetylases. NAD+ production in cells are regulated by several pathways: the rate limiting enzyme in NAD+ production through salvage pathways is NAMPT, whose expression is under the 
control of the BMAL1/CLOCK transcriptional complex; the rhythmic NAMPT expression contributes to the daily oscillation of NAD+ concentration [78, 79]. In turn, SIRT1 deacetylates PER and BMAL1 proteins, providing feedback regulation to the clock [80,81]. Whether the circadian clock contributes to known induction of NAD+ level upon CR needs to be studied.

Several groups have recently reported circadian regulation of the mTOR signaling pathway $[28,82$, 83]. Rhythms in mTORC1 activity were observed in the SCN and in metabolic tissues such as the liver. These rhythms may contribute to the rhythms in protein biosynthesis, temperature regulation or organism response to infection. BMAL1 was reported as a negative regulator of mTORC1 activity [84]; therefore, one might expect that increased BMAL1 activity may contribute to the reduction of mTORC1 activity upon calorie restriction, however, it was never formally studied.

Finally, calorie restriction increases cell resistance to various stress including oxidative stress. Circadian clock has been established as an important regulator of ROS homeostasis [85, 86]. Concentrations of ROS and many products of oxidation display circadian rhythms in blood and tissues in vivo. Circadian clock mutants have increased level of ROS and oxidative damage, supporting the importance of clock mechanisms in the oxidative stress response $[85,86]$. Several factors may contribute to the circadian clockdependent regulation of ROS homeostasis. Circadian clock-controlled hormone melatonin is a well-known antioxidant; the master regulator of oxidative stress response transcriptional factor $\mathrm{NrF} 2$ is under the direct circadian clock transcriptional control through BMAL1 [87].

Calorie restriction requires a significant level of optimization; indeed, an organism has to adapt to reduced level of resources. In order to maintain homeostasis, tissues also need to synchronize their metabolic activity and produce enzymes necessary for metabolic reaction in a timely manner. The circadian clocks provide mechanisms of metabolic optimization. Indeed, as it was discussed above, the clocks control the expression and activity of many rate-limited enzymes; therefore, the clocks guarantee that metabolic reaction will go with an appropriate rate at the appropriate time. The circadian clocks enable anticipation of daily events and also confer the considerable advantage by the efficient use of energy.

\section{Conclusions}

Aging of the world population opens novel challenges for the modern biomedical science: how to improve the quality of life for aged populations, how to reduce the rate of age-associated diseases and ultimately increase longevity. Dietary paradigms have been known as modulators of aging for a long period of time [60]. There is evidence that some of the dietary paradigms such as calorie restriction extend longevity in many organisms [60]. Multiple signaling pathways are proposed as mediators of the beneficial effects of diets on health, and recent data suggest that circadian clocks can be among the important players. The importance of the interplay between the feeding cycles and clocks in humans is an emerging field. Multiple studies on experimental or work-related circadian misalignment provide data on metabolic and health effects. Restoration of the rhythms (pharmacologically, through the light therapy or the timed feeding) may be beneficial in the clinical setting and for the treatment of mood-related disorders [88, 89]. Thus, targeting the circadian clocks through feeding or pharmacological intervention opens a novel direction in anti-aging therapy and warrants further study on the interplay between the diets, the clocks, and longevity.

\section{Acknowledgments}

This work was supported by R01AG039547 and funds from GRHD center from CSU to RVK.

\section{References}

[1] Bell-Pedersen D, Cassone VM, Earnest DJ, Golden SS, Hardin PE, Thomas TL, Zoran MJ. Circadian rhythms from multiple oscillators: Lessons from diverse organisms. Nat Rev Genet. 2005;6(7):544.

[2] Green CB, Takahashi JS, Bass J. The meter of metabolism. Cell. 2008;134(5):728.

[3] Dardente H, Cermakian N. Molecular circadian rhythms in central and peripheral clocks in mammals. Chronobiol Int. 2007;24(2): 195 .

[4] Curtis AM, Fitzgerald GA. Central and peripheral clocks in cardiovascular and metabolic function. Ann Med. 2006;38(8):552.

[5] Davis S, Mirick DK, Stevens RG. Night shift work, light at night, and risk of breast cancer. J Natl Cancer Inst. 2001;93(20): 1557 .

[6] Fujino Y, Iso H, Tamakoshi A, Inaba Y, Koizumi A, Kubo T, Yoshimura T. A prospective cohort study of shift work and 
risk of ischemic heart disease in Japanese male workers. Am J Epidemiol. 2006;164(2):128.

[7] Yu EA, Weaver DR. Disrupting the circadian clock: Genespecific effects on aging, cancer, and other phenotypes. Aging (Albany NY). 2010;3(5):479.

[8] Froy O. Circadian rhythms, aging, and life span in mammals. Physiol. 2011;26(4):225.

[9] Niccoli T, Partridge L. Ageing as a risk factor for disease. Curr Biol. 2012;22(17):R741.

[10] Kondratova AA, Kondratov RV. The circadian clock and pathology of the ageing brain. Nat Rev Neurosci. 2012;13(5):325.

[11] Reilly DF, Westgate EJ, FitzGerald GA. Peripheral circadian clocks in the vasculature. Arter Thromb Vasc Biol. 2007;27(8): 1694 .

[12] Levi F, Focan C, Karaboue A, de la Valette V, Focan-Henrard D, Baron B, Kreutz F, Giacchetti S. Implications of circadian clocks for the rhythmic delivery of cancer therapeutics. Adv Drug Deliv Rev. 2007;59(9-10):1015.

[13] Fonseca Costa SS, Ripperger JA. Impact of the circadian clock on the aging process. Frontiers in Neurology. 2015;6:43.

[14] Froy O, Chapnik N, Miskin R. Relationship between calorie restriction and the biological clock: Lessons from long-lived transgenic mice. Rejuvenation Res. 2008;11(2):467.

[15] Khapre RV, Samsa WE, Kondratov RV. Circadian regulation of cell cycle: Molecular connections between aging and the circadian clock. Ann Med. 2010;42(6):404.

[16] Colwell CS. Linking neural activity and molecular oscillations in the SCN. Nat Rev Neurosci. 2011;12(10):553.

[17] Dibner C, Schibler U, Albrecht U. The mammalian circadian timing system: Organization and coordination of central and peripheral clocks. Annu Rev Physiol. 2010;72:517.

[18] Schibler U, Sassone-Corsi P. A web of circadian pacemakers. Cell. 2002;111(7):919.

[19] Ko CH, Takahashi JS. Molecular components of the mammalian circadian clock. Hum Mol Genet. 2006;15 Spec No:R271.

[20] Harms E, Kivimae S, Young MW, Saez L. Posttranscriptional and posttranslational regulation of clock genes. J Biol Rhythm. 2004;19(5):361.

[21] Panda S, Hogenesch JB. It's all in the timing: Many clocks, many outputs. J Biol Rhythm. 2004;19(5):374.

[22] Silver R, Balsam PD, Butler MP, LeSauter J. Food anticipation depends on oscillators and memories in both body and brain. Physiol Behav. 2011;104(4):562.

[23] Mistlberger RE. Neurobiology of food anticipatory circadian rhythms. Physiol Behav. 2011;104(4):535.

[24] Davidson AJ. Lesion studies targeting food-anticipatory activity. European Journal of Neuroscience. 2009;30: 1658.

[25] Challet E. Interactions between light, mealtime and calorie restriction to control daily timing in mammals. J Comp Physiol B. 2010;180(5):631.

[26] Feillet CA, Ripperger JA, Magnone MC, Dulloo A, Albrecht $\mathrm{U}$, Challet E. Lack of food anticipation in per2 mutant mice. Curr Biol. 2006;16(20):2016.

[27] Mistlberger RE, Buijs RM, Challet E, Escobar C, Landry GJ, Kalsbeek A, Pevet P, Shibata S. Food anticipation in Bmal1/- and AAV-Bmal1 rescued mice: A reply to Fuller et al. J Circadian Rhythms. 2009;7:11.
[28] Khapre RV, Patel S, Kondratova AA, Chauhary A, Vilingkaar $\mathrm{N}$, Antoch M, Kondratov RV. Metabolic clock generates nutrient anticipation rhythms in mTOR signaling. Aging (Albany NY). 2014;6(8):675.

[29] Damiola F, Le Minli N, Preitner N, Kornmann B, FleuryOlela F, Schibler U. Restricted feeding uncouples circadian oscillators in peripheral tissues from the central pacemaker in the suprachiasmatic nucleus. Genes Dev. 2000;14(23):2950.

[30] Stokkan KA, Yamazaki S, Tei H, Sakaki Y, Menaker M. Entrainment of the circadian clock in the liver by feeding. Science. 2001;291(5503):490.

[31] Kohsaka A, Laposky AD, Ramsey KM, Estrada C, Joshu C, Kobayashi Y, Turek FW, Bass J. High-fat diet disrupts behavioral and molecular circadian rhythms in mice. Cell Metab. 2007;6(5):414.

[32] Bass J, Takahashi JS. Circadian integration of metabolism and energetics. Science. 2011;330(6009):1349.

[33] Sherman H, Genzer Y, Cohen R, Chapnik N, Madar Z, Froy $\mathrm{O}$. Timed high-fat diet resets circadian metabolism and prevents obesity. FASEB J. 2012;26(8):3493.

[34] Hatori M, Vollmers C, Zarrinpar A, DiTacchio L, Bushong EA, Gill S, Leblanc M, Chaix A, Joens M, Fitzpatrick JA, Ellisman MH, Panda S. Time-restricted feeding without reducing caloric intake prevents metabolic diseases in mice fed a high-fat diet. Cell Metab. 2012;15(6):848.

[35] Gill S, Panda S. A smartphone app reveals erratic diurnal eating patterns in humans that can be modulated for health benefits. Cell Metab. 2015;22(5):789.

[36] Challet E, Solberg LC, Turek FW. Entrainment in calorierestricted mice: Conflicting zeitgebers and free-running conditions. Am J Physiol. 1998;274(6 Pt 2):R1751.

[37] Taormina G, Mirisola MG. Calorie restriction in mammals and simple model organisms. Biomed Res Int. 2014;2014:308690.

[38] Katewa SD, Akagi K, Bose N, Rakshit K, Camarella T, Zheng X, Hall D, Davis S, Nelson CS, Brem RB, Ramanathan A, Sehgal A, Giebultowicz JM, Kapahi P. Peripheral circadian clocks mediate dietary restriction-dependent changes in lifespan and fat metabolism in drosophila. Cell Metabolism. 2015;23(1):143.

[39] Patel SA, Chaudhari A, Gupta R, Velingkaar N, Kondratov RV. Circadian clocks govern calorie restriction-mediated life span extension through BMAL1- and IGF-1-dependent mechanisms. FASEB J. 2016;30(4):1634.

[40] Mendoza J, Drevet K, Pévet P, Challet E. Daily meal timing is not necessary for resetting the main circadian clock by calorie restriction. J Neuroendocrinol. 2008;20(2): 251

[41] Vollmers C, Gill S, DiTacchio L, Pulivarthy SR, Le HD, Panda S. Time of feeding and the intrinsic circadian clock drive rhythms in hepatic gene expression. Proc Natl Acad Sci U S A. 2009;106(50):21453.

[42] Tahara Y, Otsuka M, Fuse Y, Hirao A, Shibata S. Refeeding after fasting elicits insulin-dependent regulation of Per2 and Rev-erb $\alpha$ with shifts in the liver clock. J Biol Rhythms. 2011;26(3):230.

[43] Yamajuku D, Inagaki T, Haruma T, Okubo S, Kataoka Y, Kobayashi S, Ikegami K, Laurent T, Kojima T, Noutomi $\mathrm{K}$, Hashimoto S, Oda H. Real-time monitoring in threedimensional hepatocytes reveals that insulin acts as a synchronizer for liver clock. Sci Rep. 2012;2:439. 
[44] Oike H, Oishi K, Kobori M. Nutrients, clock genes, and chrononutrition. Curr Nutr Rep. 2014;3:204.

[45] Hirota T, Kon N, Itagaki T, Hoshina N, Okano T, Fukada Y. Transcriptional repressor TIEG1 regulates Bmall gene through GC box and controls circadian clockwork. Genes to Cells. 2010;15(2):111.

[46] Iwanaga H, Yano M, Miki H, Okada K, Azama T, Takiguchi S, Fujiwara Y, Yasuda T, Nakayama M, Kobayashi M, Oishi K, Ishida N, Nagai K, Monden M. Per2 gene expressions in the suprachiasmatic nucleus and liver differentially respond to nutrition factors in rats. JPEN J Parenter Enteral Nutr. 2005;29(3):157.

[47] Challet E, Losee-Olson S, Turek FW. Reduced glucose availability attenuates circadian responses to light in mice. Am J Physiol. 1999;276(4 pt 2):R1063.

[48] Lamia KA, Sachdeva UM, DiTacchio L, Williams EC, Alvarez JG, Egan DF, Vasquez DS, Juguilon H, Panda S, Shaw RJ, Thompson CB, Evans RM. AMPK regulates the circadian clock by cryptochrome phosphorylation and degradation. Science. 2009;326(5951):437.

[49] Li MD, Ruan H Bin, Hughes ME, Lee JS, Singh JP, Jones SP, Nitabach MN, Yang X. O-GlcNAc signaling entrains the circadian clock by inhibiting BMAL1/CLOCK ubiquitination. Cell Metab. 2013;17(2):303.

[50] Adamovich Y, Aviram R, Asher G. The emerging roles of lipids in circadian control. Biochimica et Biophysica Acta Molecular and Cell Biology of Lipids. 2015;1851(8):1017.

[51] Lipton JO, Yuan ED, Boyle LM, Ebrahimi-Fakhari D, Kwiatkowski E, Nathan A, Guttler T, Davis F, Asara JM, Sahin M. The circadian protein BMAL1 regulates translation in response to S6K1-mediated phosphorylation. Cell. 2015;161(5):1138.

[52] Laplante M, Sabatini DM. mTOR signaling in growth control and disease. Cell. 2012;149(2):274.

[53] Hirao A, Tahara Y, Kimura I, Shibata S. A balanced diet is necessary for proper entrainment signals of the mouse liver clock. PLoS One. 2009;4(9):E6909.

[54] Oike H, Nagai K, Fukushima T, Ishida N, Kobori M. Feeding cues and injected nutrients induce acute expression of multiple clock genes in the mouse liver. PLoS One. 2011;6(8):E23709.

[55] Oike H, Kobori M, Suzuki T, Ishida N. Caffeine lengthens circadian rhythms in mice. Biochem Biophys Res Commun. 2011;410(3):654.

[56] Oike H, Kobori M. Resveratrol regulates circadian clock genes in Rat-1 fibroblast cells. Biosci Biotechnol Biochem. 2008;72(11):3038.

[57] Oike H, Nagai K, Fukushima T, Ishida N, Kobori M. High-salt diet advances molecular circadian rhythms in mouse peripheral tissues. Biochem Biophys Res Commun. 2010;402(1):7.

[58] Zarrinpar A, Chaix A, Panda S. Daily eating patterns and their impact on health and disease. Trends Endocrinol Metab. 2016;27(2):69.

[59] Mukherji A, Kobiita A, Chambon P. Shifting the feeding of mice to the rest phase creates metabolic alterations, which, on their own, shift the peripheral circadian clocks by 12 hours. Proc Natl Acad Sci. 2015;112(48):E6691.

[60] Piper MD, Bartke A. Diet and aging. Cell Metab. 2008;8(2):99.

[61] Brown-Borg HM. Hormonal regulation of longevity in mammals. Ageing Res. 2007;6(1):28.
[62] Blagosklonny MV. Aging: ROS or TOR. Cell Cycle. 2008;7(21):3344.

[63] Guarente L, Picard F. Calorie restriction-the SIR2 connection. Cell. 2005;120(4):473.

[64] Kalsbeek A, La Fleur S, Fliers E. Circadian control of glucose metabolism. Vol. 3, Molecular Metabolism. 2014; 3(4):372.

[65] Marcheva B, Ramsey KM, Buhr ED, Kobayashi Y, Su H, Ko $\mathrm{CH}$, Ivanova G, Omura C, Mo S, Vitaterna MH, Lopez JP, Philipson LH, Bradfield CA, Crosby SD, JeBailey L, Wang X, Takahashi JS, Bass J. Disruption of the clock components CLOCK and BMAL1 leads to hypoinsulinaemia and diabetes. Nature. 2010;466(7306):627.

[66] Zhao Y, Zhang Y, Zhou M, Wang S, Hua Z, Zhang J. Loss of $\mathrm{mPer} 2$ increases plasma insulin levels by enhanced glucosestimulated insulin secretion and impaired insulin clearance in mice. FEBS Lett. 2012;586(9):1306.

[67] Barclay JL, Shostak A, Leliavski A, Tsang AH, Johren O, Muller-Fielitz H, Landgraf D, Naujokat N, van der Horst GT, Oster H. High-fat diet-induced hyperinsulinemia and tissue-specific insulin resistance in Cry-deficient mice. Am J Physiol Endocrinol Metab. 2013;304(10):E1053.

[68] Rudic RD, McNamara P, Curtis AM, Boston RC, Panda S, Hogenesch JB, FitzGerald GA. BMAL1 and CLOCK, two essential components of the circadian clock, are involved in glucose homeostasis. PLoS Biol. 2004;2(11):E377.

[69] Zhang EE, Liu Y, Dentin R, Pongsawakul PY, Liu AC, Hirota T, Nusinow DA, Sun X, Landais S, Kodama Y, Brenner DA, Montminy M, Kay SA. Cryptochrome mediates circadian regulation of cAMP signaling and hepatic gluconeogenesis. Nat Med. 2010;16(10):1152.

[70] Lamia KA, Papp SJ, Yu RT, Barish GD, Uhlenhaut NH, Jonker JW, Downes M, Evans RM. Cryptochromes mediate rhythmic repression of the glucocorticoid receptor. Nature. 2011;480(7378):552.

[71] Zani F, Breasson L, Becattini B, Vukolic A, Montani JP, Albrecht U, Provenzani A, Ripperger JA, Solinas G. PER2 promotes glucose storage to liver glycogen during feeding and acute fasting by inducing Gys2 PTG and GL expression. Mol Metab. 2013;2(3):292.

[72] Gooley Joshua J, Eric Chern-Pin Chua. Diurnal regulation of lipid metabolism and applications of circadian lipidomics. Journal of Genetics and Genomics. 2014;41(5):231.

[73] Kumar Jha P, Challet E, Kalsbeek A. Circadian rhythms in glucose and lipid metabolism in nocturnal and diurnal mammals. Molecular and Cellular Endocrinology. 2015;418(1):74.

[74] Shimba S, Ishii N, Ohta Y, Ohno T, Watabe Y, Hayashi M, Wada T, Aoyagi T, Tezuka M. Brain and muscle Arnt-like protein-1 (BMAL1), a component of the molecular clock, regulates adipogenesis. Proc Natl Acad Sci. 2005;102(34): 12071.

[75] Guo B, Chatterjee S, Li L, Kim JM, Lee J, Yechoor VK, Minze LJ, Hsueh W, Ma K. The clock gene, brain and muscle Arnt-like 1, regulates adipogenesis via Wnt signaling pathway. FASEB J. 2012;26(8):3453.

[76] Douris N, Kojima S, Pan X, Lerch-Gaggl AF, Duong SQ, Hussain MM, Green CB. Nocturnin regulates circadian trafficking of dietary lipid in intestinal enterocytes. Curr Biol. 2011;21(16):1347.

[77] Shea SA, Hilton MF, Orlova C, Timothy Ayers R, Mantzoros CS. Independent circadian and sleep/wake regulation 
of adipokines and glucose in humans. J Clin Endocrinol Metab. 2005;90(5):2537.

[78] Ramsey KM, Yoshino J, Brace CS, Abrassart D, Kobayashi Y, Marcheva B, Hong HK, Chong JL, Buhr ED, Lee C, Takahashi JS, Imai S, Bass J. Circadian clock feedback cycle through NAMPT-mediated NAD+ biosynthesis. Science. 2009;324(5927):651.

[79] Nakahata Y, Sahar S, Astarita G, Kaluzova M, SassoneCorsi P. Circadian control of the NAD+ salvage pathway by CLOCK-SIRT1. Science. 2009;324(5927):654.

[80] Nakahata Y, Kaluzova M, Grimaldi B, Sahar S, Hirayama J, Chen D, Guarente LP, Sassone-Corsi P. The NAD+dependent deacetylase SIRT1 modulates CLOCK-mediated chromatin remodeling and circadian control. Cell. 2008; 134(2):329.

[81] Asher G, Gatfield D, Stratmann M, Reinke H, Dibner C, Kreppel F, Mostoslavsky R, Alt FW, Schibler U. SIRT1 regulates circadian clock gene expression through PER2 deacetylation. Cell. 2008;134(2):317.

[82] Jouffe C, Cretenet G, Symul L, Martin E, Atger F, Naef F, Gachon F. The circadian clock coordinates ribosome biogenesis. PLoS Biol. 2013;11(1):E1001455.

[83] Cornu M, Oppliger W, Albert V, Robitaille AM, Trapani F, Quagliata L, Fuhrer T, Sauer U, Terracciano L, Hall MN. Hepatic mTORC1 controls locomotor activity, body temperature, and lipid metabolism through FGF21. Proc Natl Acad Sci. 2014;111:11592.

[84] Khapre RV, Kondratova AA, Patel S, Dubrovsky Y, Wrobel M, Antoch MP, Kondratov R V. BMAL1-dependent regulation of the mTOR signaling pathway delays aging. Aging (Albany NY). 2014;6(1):48.

[85] Patel SA, Velingkaar NS, Kondratov RV. Transcriptional control of antioxidant defense by the circadian clock. Antioxid Redox Signal. 2014;20(18):2997.

[86] Hardeland R, Coto-Montes A, Poeggeler B. Circadian rhythms, oxidative stress, and antioxidative defense mechanisms. Chronobiol Int. 2003;20(6):921.

[87] Pekovic-Vaughan V, Gibbs J, Yoshitane H, Yang N, Pathiranage D, Guo B, Sagami A, Taguchi K, Bechtold D, Loudon A, Yamamoto M, Chan J, van der Horst GTJ, Fukada Y, Meng QJ. The circadian clock regulates rhythmic activation of the NRF2/glutathionemediated antioxidant defense pathway to modulate pulmonary fibrosis. Genes Dev. 2014;28:548.

[88] Lanfumey L, Mongeau R, Hamon M. Biological rhythms and melatonin in mood disorders and their treatments. Vol. 138, Pharmacology and Therapeutics. 2013;138(2):176.

[89] Sunderram J, Sofou S, Kamisoglu K, Karantza V, Androulakis IP. Time-restricted feeding and the realignment of biological rhythms: Translational opportunities and challenges. J Transl Med. 2014;12(1):79. 